\title{
Effective medium theory for the conductivity of disordered metallic nanowire networks
}

\author{
Colin O'Callaghan* ${ }^{1,2,3}$ Claudia Gomes da Rocha, ${ }^{1,2,3}$ Hugh G. \\ Manning, ${ }^{4,2,3}$ John J. Boland, ${ }^{4,2,3}$ and Mauro S. Ferreira ${ }^{1,2,3}$ \\ ${ }^{1}$ School of Physics, Trinity College Dublin, Dublin 2, Ireland \\ ${ }^{2}$ Centre for Research on Adaptive Nanostructures and Nanodevices (CRANN), \\ Trinity College Dublin, Dublin 2, Ireland \\ ${ }^{3}$ AMBER Research Centre, Trinity College Dublin, Dublin 2, Ireland:* \\ ${ }^{4}$ School of Chemistry, Trinity College Dublin, Dublin 2, Ireland
}

\begin{abstract}
Motivated by numerous technological applications, there is current interest in the study of the conductive properties of networks made of randomly dispersed nanowires. The sheet resistance of such networks is normally calculated by numerically evaluating the conductance of a system of resistors but due to disorder and with so many variables to account for, calculations of this type are computationally demanding and may lack mathematical transparency. Here we establish the equivalence between the sheet resistance of disordered networks and that of a regular ordered network. Rather than through a fitting a scheme, we provide a recipe to find the effective medium network that captures how the resistance of a nanowire network depends on several different parameters such as wire density, electrode size and electrode separation. Furthermore, the effective medium approach provides a simple way to distinguish the sheet resistance contribution of the junctions from that of the nanowires themselves. The contrast between these two contributions determines the potential to optimize the network performance for a particular application.
\end{abstract}

*Electronic address: ocallaco@tcd.ie 


\section{INTRODUCTION}

Randomly dispersed nanowires networks (NWN) are flexible, electrically active materials with great promise for use as transparent conductors [1]3], thin-film solar cells [4] 6 , and sensor devices [7, 8]. NWNs are most typically comprised of metallic nanowires, of which each wire is coated with either a surface functionalisation or oxide passivation layer to facilitate solution phase processing by preventing flocculation. The exploitation of NWNs for any of these applications involves the activation or switching on of the junctions between wires in the network, which is typically accomplished by using heat [9, 10], pressure [1] and electrical stressing[12] to yield a material with definitive properties, e.g., sheet resistance and transparency.

Fig. 1(a) shows an SEM image of a typical NWN, where hundreds of high aspect-ratio wires are randomly deposited onto an insulating substrate and contacted by two metallic electrodes on opposite ends of the image. Whilst such NWN devices may require no precise spatial ordering, accurately predicting the physical responses of such a system is challenging due to the large uncertainties caused by two main types of disorder: the randomness with which wires are spatially distributed and the inherent fluctuations on the individual characteristics of the wires. This calls for averaging strategies that reduce the impact of these fluctuations in any calculations. With that in mind, we have recently introduced a method that processes SEM microscopy images of NWNs and captures the precise locations of all wires of a given sample[13]. This establishes the exact connectivity the NWN possesses and removes the need for averaging over the wire locations, consequently reducing the fluctuations induced by spatial disorder.

This image-processing technique is a welcome tool to improve the descriptive power of simulations but the study of the electrical response of these films remains challenging because it also depends on a multitude of other factors such as material type, wire length and diameter, interwire contact quality, wire density, network connectivity, etc. It is frequently assumed to facilitate calculations of this type that the overall network resistance is dominated by the junction resistances formed between adjacent wires of the network[14]. However, calculated junction resistances based on this assumption have now been shown to be orders of magnitude higher than those subsequently measured[15], indicating that the internal contribution of the individual nanowires cannot be neglected and is one more factor to be 

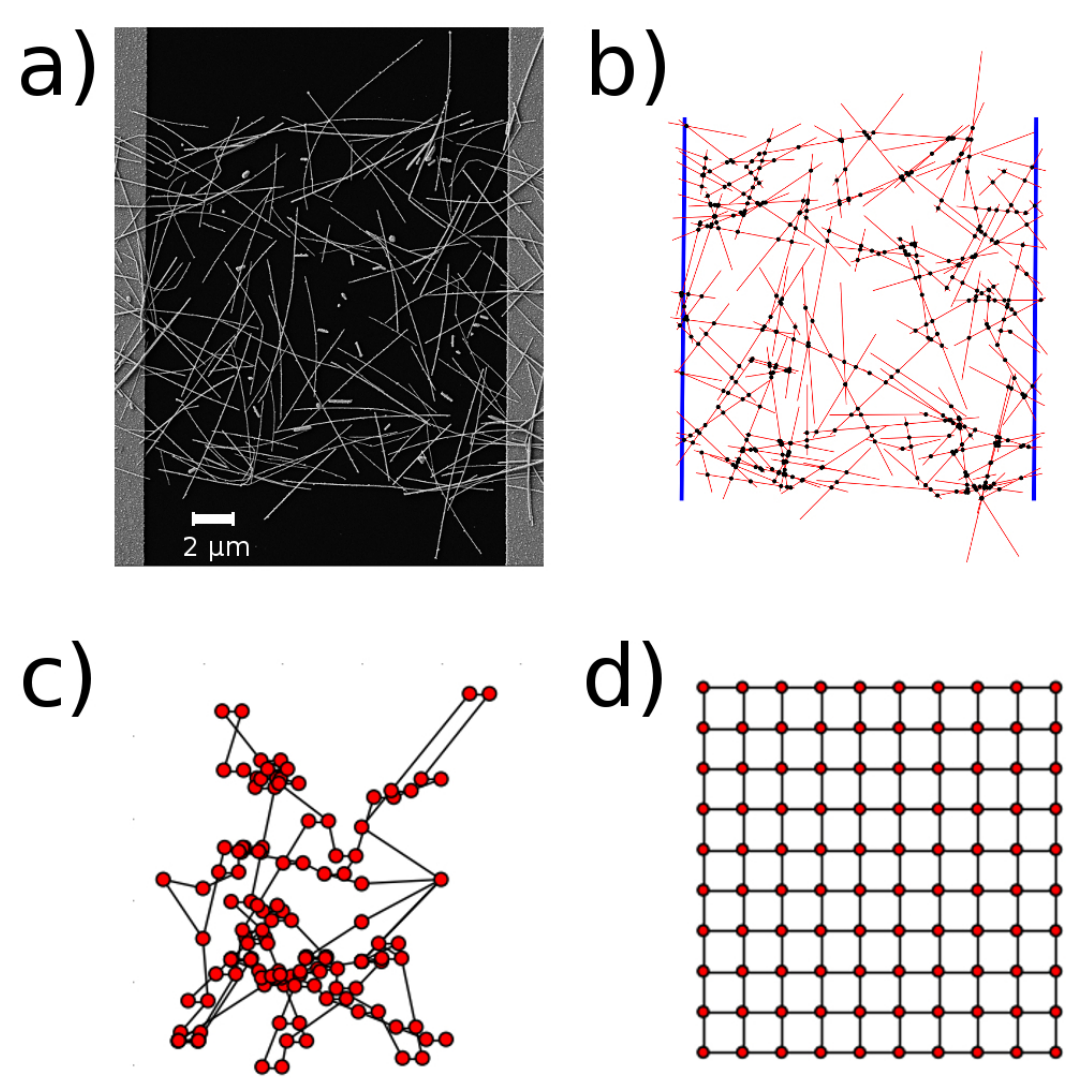

FIG. 1: (a) SEM micrograph image of a Ag-NWN with hundreds of wires randomly distributed on top of an insulating substrate. Two electrodes on both sides of the sample, shown as vertical gray bars, are connected by numerous paths formed by the wires. (b) After the image is processed, the digitized version of the image records each wire location and provides full information about the intersection points of each wire; (c) Mathematical graph showing voltage nodes as points and connecting resistors as edges; (d) The simplified graph of a square lattice representing a regular ordered network.

accounted for. With so many ingredients affecting the sheet resistance of these films, a closed-form expression for the conductance of such heavily disordered networks would be very welcome.

At present, there is no theoretical description based on real-world NWNs in which their far-from-perfect physical characteristics are accounted for in a closed-form mathematical representation. This is typically done by means of laborious Monte-Carlo procedures used to determine universal behaviours of simplified computer-generated NWNs [16 19]. Indeed, such techniques are so computationally demanding that the dependence of the sheet re- 
sistance on all the possible physical characteristics of real NWN such as the wire density, material properties, connectivity, etc, can only be estimated numerically. However, closedform analytical expressions for the conductance of ordered homogeneous networks are known [20]. These are spatially ordered networks (e.g. square, triangular, hexagonal, etc) connected by identical resistors throughout. Whether these expressions can be of use to describe heavily disordered structures, even though they are far from ordered and homogenous, is the question posed here. In this manuscript we show that by mapping the disordered structures onto a corresponding effective medium, we can obtain the sheet resistance of NWN with an arbitrarily large density of wires. Further manipulation of these expressions enables us to describe the conductivity of these films under real experimental conditions. In fact, we show that dense networks composed of nanowires of non-uniform lengths and diameters contacted by finite-sized electrodes can be fully described by this approach. Furthermore, we argue that not only can we reproduce experimental measurements but we are also able to search for optimization conditions that will minimize the sheet resistance of a NWN.

The sequence adopted in this paper is as follows. For the sake of completeness, we start by writing the equivalent resistance (or conductance) of a network comprised of identical resistors forming an infinitely large regular ordered lattice. Because Kirchhoff's laws on networks are expressed in terms of a Laplacian matrix, it is useful to solve this problem using Green Function (GF) methods. Indeed, writing it in terms of GF becomes extremely convenient when disorder is included since there is a vast body of knowledge on solving Laplacian-like equations [20] for disordered matrices[21, 22]. We then present the effective medium theory (EMT) that allows us to express the problem of calculating the conductance of a inhomogeneous disordered network in terms of an ordered regular network that has a homogeneous resistance 23. Without any fitting parameter, we are able to demonstrate that this closed-form expression for the conductance provides an excellent match both with simulations and with experimental results. We conclude by illustrating how this approach can be useful in the study of NWNs as well as in other disordered materials.

\section{THEORETICAL METHOD}

We start this section by describing how the NWN depicted in Fig. 1(a) is transformed into a resistor network. Fig. 1(b) shows the digitized version of the original image on the top 
left panel and contains full information about the position, orientation and the intersection points of all wires [13]. Each intersection point is mapped onto a voltage node connected by resistors, the value of which depends on whether the nodes it connects are on the same wire or on different ones. In the case of nodes on different wires, a resistance is assigned according to the distribution of measured individual junction resistances recently reported [15]. In the case of neighbouring nodes on the same wire, the corresponding resistance value assigned depends on the wire resistivity and is simply proportional to the length of the wire section connecting the nodes. It is worth mentioning that this length distribution is different from the wire length distribution and simply results from the randomness of the intersection points on all wires, i.e., it follows a Poisson distribution. While it is not so difficult to visualize this network for a small number of wires, it becomes far too complex for any reasonably sized NWN. Representing the network by a mathematical graph that depicts voltage nodes as points and connecting resistors as edges, as illustrated in Fig. 1)(c), one can see how complex the visualization becomes in the case of high-density NWN. A considerably simpler graph is shown on Fig. 1(d) where a regular homogeneous network is represented by a square lattice. By establishing the conditions under which the networks of Figs. 1(c) and 1(d) have similar responses, we can find closed-form expressions that describe the sheet resistance of films like the ones shown in Fig. 1(a). In what follows, we consider the case of a regular homogeneous network with external current injected and extracted on two nodes a certain distance apart, for which closed-form expressions can be easily obtained.

\section{A. Inter-node resistance of a regular network}

By using Kirchhoff's and Ohms laws, the inter-node Resistance Green's Function $(\mathrm{RGF})[20$ is ideal to obtain the equivalent conductance of regular networks. In the case of infinitely large networks represented by e.g. square, hexagonal and triangular lattices, closed-form expressions give rise to simple analytical expressions that describe the equivalent resistance between any two points of the network a certain distance apart. We summarise a derivation of this expression in this section as it also illustrates what is needed to deal with disordered finite networks in the presence of extended electrodes, which is more closely related to the realistic experimental setup of NWN films.

The RGF describes the resistance between two nodes on an infinite regular lattice sepa- 
rated by the vector $\vec{r}$. We shall consider a square circuit lattice, as shown in Fig. 1(d). Thus the position vector of nodes is given by $\vec{r}_{\ell_{1}, \ell_{2}}=\ell_{1} \vec{a}_{1}+\ell_{2} \vec{a}_{2}, \vec{a}_{i}$ are the primitive vectors of the square lattice and $\ell_{i} \in \mathbb{Z}$. The primitive vectors $\vec{a}_{i}$ have reciprocal lattice vectors $\vec{k}_{i}$. We set $\left|\vec{a}_{i}\right|=1$ for simplicity. Resistors, the edges connecting nodes in the lattice, are assumed to have the same value $R$. In order to obtain the resistance between two nodes in the network, a current $I$ is injected to a node at position $\vec{r}$ and extracted at point $\vec{r}^{\prime}$. The potential at the site $\vec{r}$ is $V(\vec{r})$. From Kirchhoff's and Ohm's laws:

$$
I(\vec{r}) R=\sum_{\vec{n}}[V(\vec{r})-V(\vec{r}+\vec{n})]
$$

$\vec{n}$ are the vectors connecting $\vec{r}$ to its nearest neighbours. The right hand side can be described using the lattice Laplacian,

$$
-\Delta_{\vec{r}} f(\vec{r})=\sum_{\vec{n}}[f(\vec{r})-f(\vec{r}+\vec{n})]
$$

which leads to

$$
\Delta_{\vec{r}} V(\vec{r})=-I(\vec{r}) R
$$

Let the current be injected at the origin and extracted at an arbitrary site $\vec{r}_{0}$. Thus $I(\vec{r})$ can be written as $I(\vec{r})=I\left(\delta_{\vec{r}, 0}-\delta_{\vec{r}, \vec{r}_{0}}\right)$, where $\delta_{x, y}$ is the Kronecker delta function. Bringing this together the resistance between the two nodes is:

$$
R_{n}\left(\vec{r}_{0}\right)=\frac{V(\overrightarrow{0})-V\left(\vec{r}_{0}\right)}{I}
$$

We introduce a lattice Green's function and define it as

$$
\Delta_{\left(\vec{r}^{\prime}\right)} G\left(\vec{r}-\vec{r}^{\prime}\right)=-\delta_{\vec{r}, \vec{r}^{\prime}}
$$

Eq. (3) is a Poisson-type equation and can be solved using the lattice GF, which is defined simply as the inverse of the Laplacian matrix $\Delta[24]$,

$$
V(\vec{r})=R \sum_{\vec{r}^{\prime}}\left[G\left(\vec{r}-\vec{r}^{\prime}\right) I\left(\vec{r}^{\prime}\right)\right]=R\left[G(\vec{r}-\overrightarrow{0})-G\left(\vec{r}-\vec{r}_{0}\right)\right]
$$

Combining Eqs. (4) and (6), and the fact that the lattice GF is even one finds

$$
R_{n}\left(\vec{r}_{0}\right)=2 R\left[G(\overrightarrow{0})-G\left(\vec{r}_{0}\right)\right]
$$

Up to this point, nothing specific about the network size has been mentioned. In fact, in the case of a finite-sized network the GF definition of Eq. (5) gives rise to a square matrix 
of size $S \times S$, where $S$ is the total number of voltage nodes. In the case of infinitely large networks we make use of the symmetries of the infinite square lattice and take the Fourier transform of $G(\vec{r})$,

$$
G(\vec{r})=\int_{\vec{k} \in B Z} G(\vec{k}) e^{i \vec{k} \cdot \vec{r}}
$$

The integral is performed over the square lattice Brillouin Zone. Using Eq.(3) one finds

$$
G(\vec{k})=\frac{1}{2\left(2-\cos \left(k_{1}\right)-\cos \left(k_{2}\right)\right)}
$$

Substituting these equations into Eq. (7)

$$
R_{n}\left(\vec{r}_{0}\right)=R \int_{\vec{k} \in B Z} \frac{d \vec{k}}{(2 \pi)^{2}} \frac{1-e^{\vec{k} \cdot \vec{r}_{0}}}{2-\cos \left(k_{1}\right)-\cos \left(k_{2}\right)}
$$

Let $\vec{r}=l_{1} \vec{a}_{1}+l_{2}+\vec{a}_{2}$; this integral has an asymptotic form for large values of $l_{i}$,

$$
R_{n}(|\vec{r}|) \approx \frac{R}{\pi}\left(\ln |\vec{r}|+\gamma+\frac{\ln 8}{2}\right)
$$

where $\gamma=0.57721 \ldots$ is the Euler-Mascheroni constant. To verify the validity of Eq. (11), we

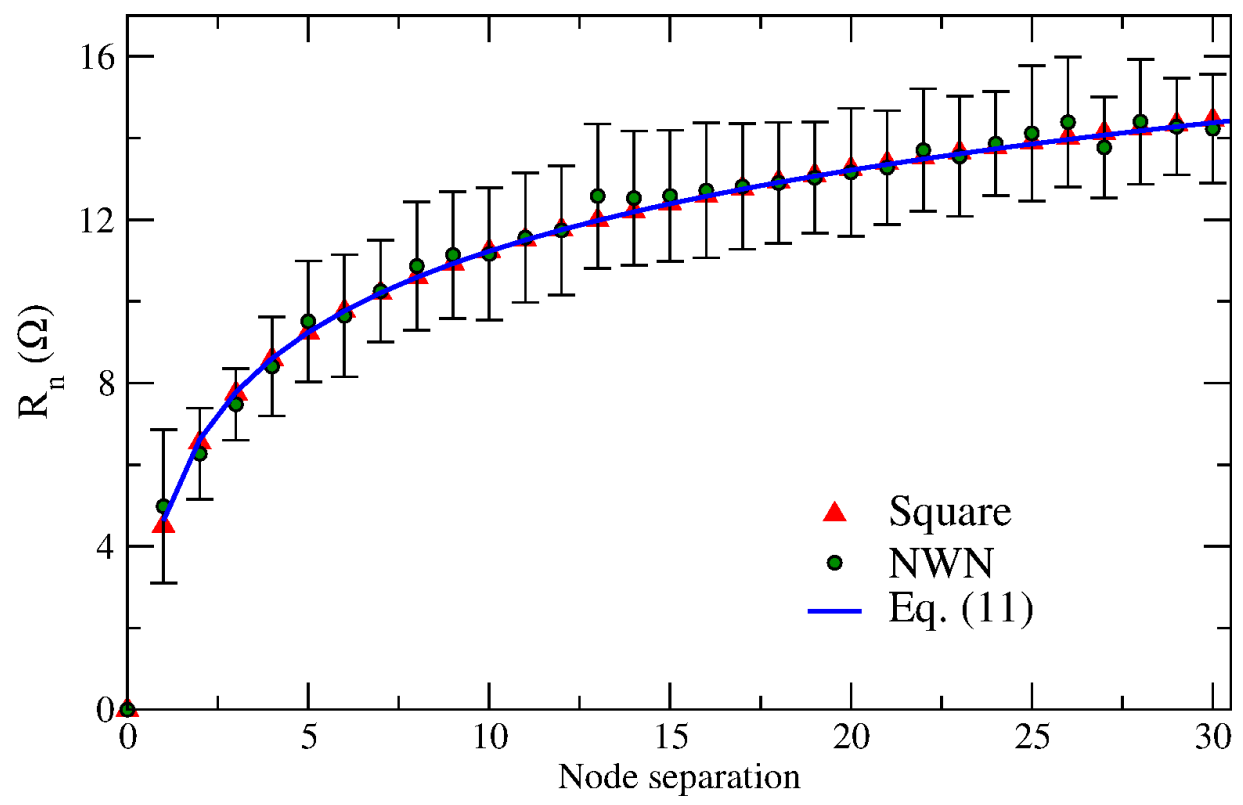

FIG. 2: Plot of the equivalent resistance $R_{n}(\vec{r})$ between two point electrodes a distance $|\vec{r}|$ apart. The solid line corresponds to the analytical expression of Eq. 11) whereas the triangular dots are the numerical calculations of $R_{n}$ for a square lattice of size $500 \times 500$. Circular dots correspond to numerical calculations of the average equivalent resistance between pairs of nodes at a given node separation on a NWN, such as one shown in Fig. 11(a).

performed a numerical calculation of the equivalent resistance for a square lattice network 
of size $500 \times 500$ nodes. The resistance was obtained by solving Kirchhoff's circuit equations written in matrix form. The resistances were probed for distinct point electrode separations and they are depicted in Fig. 2, This result shows that the Eq. (11) is indeed a good approximation for moderately large separations $|\vec{r}|$. For much larger separations finite size effects causes the numerical simulations to diverge from theory, which is not shown in the plot.

The agreement between the solid line and the triangular dots is not so surprising since both calculations are done for square-latticed networks. The latter corresponds to the numerical calculation of the equivalent resistance between two voltage nodes a distance $\vec{r}$ apart in a finite-sized network whereas the former is for infinitely large systems. The surprising feature here is the agreement shown between Eq. (11) and the circular dots of Fig. 2. These were evaluated numerically for the complex graph shown in Fig. 1(c) through a method to be described in the following sections and correspond to the realistic sample seen in Fig. 1(a). This is a clear indication that the sheet resistance of that complex NWN can be very well described by a regular network. We argue that the agreement is not coincidental and that there is always a suitable value for $R$ in Eq. (11) that reproduces the conductance behaviour of the NWN, no matter how disordered it is. It is worth mentioning that instead of a fitting scheme, our goal is to provide a simple recipe to find the effective-medium network that captures all the features seen in the realistic sample.

\section{B. Extended Electrodes embedded in a finite network}

We now wish to generalize the RGF to a system that is closer to the geometry commonly seen in experiments of this type. Rather than point electrodes embedded in infinite lattices, we now focus on the case of extended electrodes. Fig.3(a) shows the graph associated with a NWN in the presence of finite-sized electrodes represented by two separate vertical red lines. $N_{y}=7$ is the average number of wires contacted by the electrodes that are a certain distance apart. See Appendix for a description of how $N_{y}$ is calculated. Fig. 3(b) shows the equivalent homogeneous square lattice with electrodes that span the same number $N_{y}$ of resistors. Similarly to the case of point electrodes, the panel (c) shows excellent agreement between the numerical results associated with the networks shown in panels (a) and (b), confirming that the agreement is far from coincidental and reflects the similarities between 
(a)

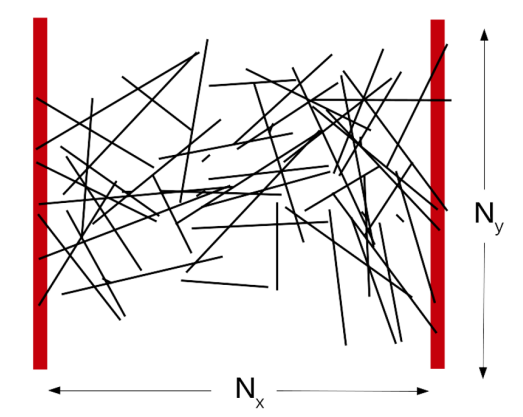

(c) (b)

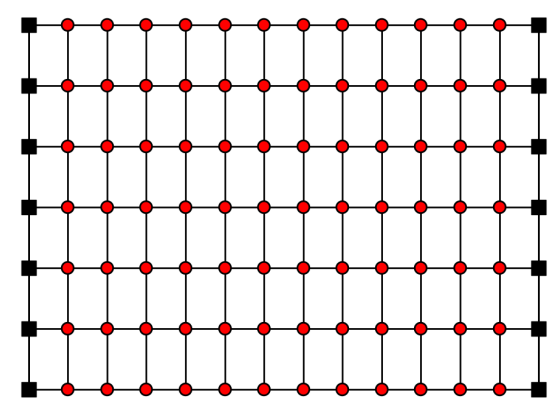

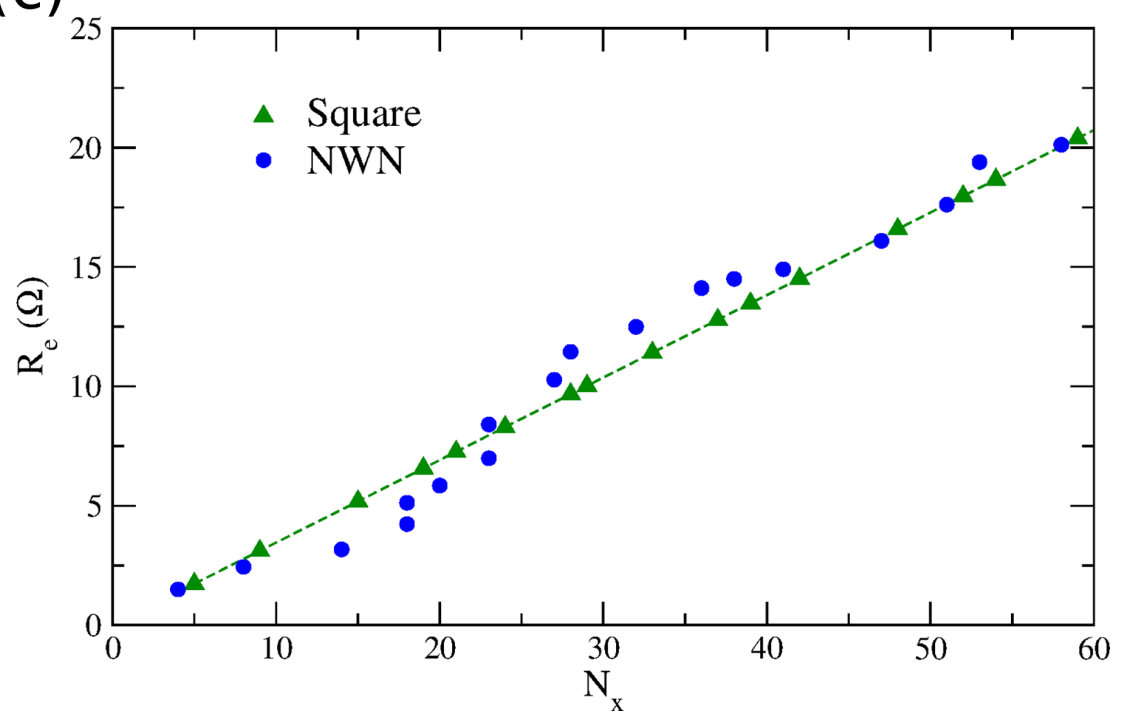

FIG. 3: (a) Digitized image of a real NWN with two separate finite-sized electrodes represented by vertical red lines. $N_{y}=7$ wires cross the electrodes. (b) Square lattice with finite-sized electrodes (represented by red dots). The number of black squares on either side corresponds to the value of $N_{y}=7$ electrode nodes obtained from panel (a). $N_{x}$ represents the characteristic number of junctions and wire segments needed to form a path between the electrodes; (c) Sheet resistance as a function of $N_{x}$. Circular dots are the calculated results for the realistic network whereas triangular dots correspond to the results of the square lattice shown in panel (b) with $R \approx 8.3 \Omega$.

the sheet resistances of both geometries. The equivalent resistance between the electrodes in Fig. 3 (b) can be expressed as 25$]$

$$
R_{e}\left(N_{x}, N_{y}\right)=R \frac{N_{x}}{N_{y}}
$$

and has a straightforward interpretation: one considers chains of $N_{x}$ resistors of resistance $\mathrm{R}$ connected in series, and there are $N_{y}$ of these chains connected in parallel.

Whilst the values of $N_{x}$ and $N_{y}$ are obtained by purely geometric arguments, shown in Appendix $\mathrm{A}$, the quantity $R$ is the key quantity to describe the sheet resistance of a NWN. As previously seen in Figs. 2 and 3, a suitable choice for the value of $R$ makes the 
regular-network results coincide with those associated with disordered NWN. Rather than a fortunate coincidence or even a fitting scheme, we can obtain this value from first principles, as we demonstrate next.

Consider a circuit lattice where the individual edge conductance, represented by the quantity $g$, follows some distribution $f(g)$. If all conductors are replaced with the effective conductance, the average electrical behaviour of the network is maintained [23]. The effective conductance $g_{m}$ can be calculated for a square circuit lattice using the following equation,

$$
\int d g f(g)\left(\frac{g_{m}-g}{g+g_{m}}\right)=0
$$

The solution to this integral equation will therefore provide the effective value of $g_{m}$ and consequently the value of $R=1 / g_{m}$ as seen in Figs. 2 and 3 . Note that this solution is crucially dependent on $f(g)$ and for that reason we need to describe well how the individual resistances connecting the neighbouring voltage nodes are distributed.

As previously mentioned, the assumption that the conductivity is dominated by the junction resistances is not always accurate and can lead to a gross overestimation of the individual resistances [14, 15. Multi-nodal representation (MNR) was recently developed in order to include the effects of intra-wire resistance on the sheet resistance of NWN [13. In the MNR scheme, network nodes are associated with intersection points on each network. The nodes are then connected with edges weighted by a wire junction conductance or an intra-wire conductance. For the case of nodes near the ends of the wires, an infinite resistor must also be included in the overall distribution to account for the two non-electrically active wire segments per wire. Therefore, a tri-conductance distribution on a square circuit lattice is used to calculate the effective resistance for a NWN.

The resistors in the NWN can be separated into 3 different categories: (i) the interwire junctions following a conductance distribution $\sigma_{j}(g)$, (ii) the inner-wire conductance following a distribution $\sigma_{i}(g)$, and (iii) non-electrically active resistors. The total number of wire segments $N_{s}$ (both current carrying segments and non-electrically active wire segments) can be written as

$$
N_{s}=2 N_{j}+N_{w}
$$

where $N_{j}$ is the total number of junctions and $N_{w}$ is the number of wires. This can be understood in the following way; The number of wire segments starts as the number of wires in the system. For every wire junction, two new wire segments are formed (one on each wire). 
The number of non-electrically active wire segments $N_{D}$ is simply $N_{D}=2 N_{w}$, i.e, two per wire. Thus the number of current carrying wire segments $N_{c}$ is $N_{c}=N_{s}-N_{D}=2 N_{j}-N_{w}$. The total number of conductors $N_{t}$ is the sum of inter-wire junctions $N_{j}$ and wire segments $N_{s}$, so $N_{t}=N_{j}+N_{s}=3 N_{j}+N_{w}$.

The probabilities for a junction conductor $\left(P_{j}\right)$, an inner conductor $\left(P_{i}\right)$ and, a nonelectrically active segment, or a "dead-end" segment, $\left(P_{d}\right)$ are given by:

$$
\begin{aligned}
P_{j} & =\frac{N_{j}}{N_{t}} \\
P_{i} & =\frac{2 N_{j}-N_{w}}{N_{t}} \\
P_{d} & =\frac{2 N_{w}}{N_{t}}
\end{aligned}
$$

Combining these expressions together, the conductance distribution to be inserted into Eq. (13) is thus:

$$
f(g)=P_{i} \sigma_{j}(g)+P_{j} \sigma_{j}(g)+P_{d} \delta(g)
$$

Bellew et al. made physical measurements of individual junction resistances and an associated resistance distribution was obtained[15], which can be seen in Fig.(4). The intra-

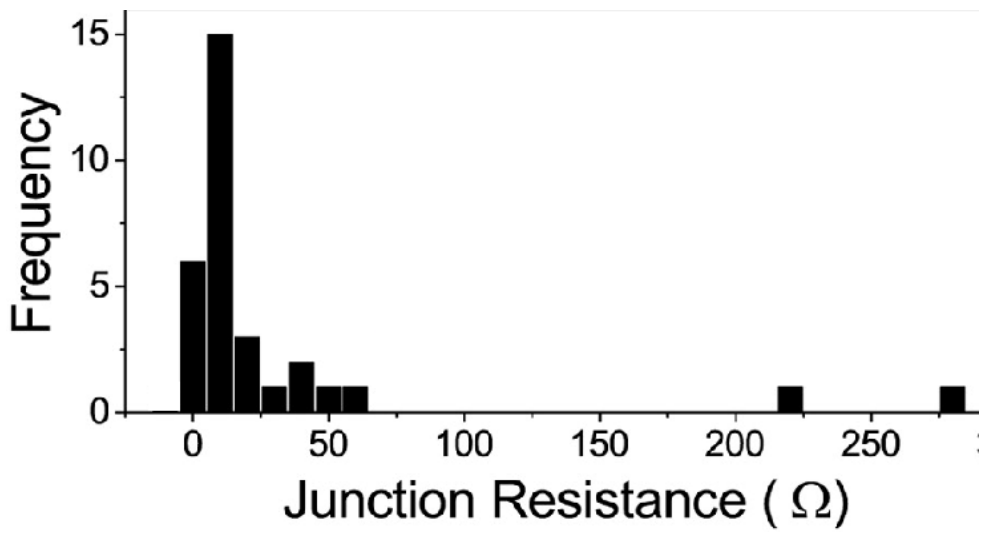

FIG. 4: Junction Resistance distribution from Bellew et al.[15]. The width of each bin in the histogram is $10 \Omega$. A peak occurs in the distribution in the $10-20 \Omega$ bin. The mean resistance occurs at approximately $11 \Omega$.

wire resistance $\left(R_{i}\right)$ is given by the usual identity relating it with its length and diameter,

$$
R_{i}=\frac{\rho l_{i}}{A}
$$

where $\rho, l_{i}, A$ are the resistivity, wire segment length and cross sectional area of the wire, respectively. Having Ag wires in mind, the values used are taken from physical measurements 
found in our recent publication [13]. The resistivity is taken as $22.6 n \Omega m$ and wire diameters are $50 \mathrm{~nm}$. The lengths of the nano-wires $(L)$ are given by a Gaussian distribution centered at $6.7 \mu \mathrm{m}$. By considering the intersection of wires as a Poisson point process one expects the segment length distribution $l_{i}$ to follow a Poisson distribution. Combining these distributions one csn construct the intra-wire conductance distribution $\sigma_{i}(g)$.

In order to simplify the calculation of the effective conductance, we replace $\sigma_{i}(g)$ and $\sigma_{j}(g)$ with their mean values $g_{i}$ and $g_{j}$ respectively. In order to calculate $g_{i}$, we must first find the average segment length $\langle l\rangle$. This is calculated by dividing the total length of all wires by the number of wire segments,

$$
<l>=\frac{N_{w} L}{N_{s}}=\frac{N_{w} L}{2 N_{j}+N_{w}} .
$$

This is combined with the experimental parameters given above. The conductance distribution then becomes:

$$
f(g)=P_{i} \delta\left(g-g_{i}\right)+P_{j} \delta\left(g-g_{j}\right)+P_{d} \delta(g)
$$

Applying this expression to Eq. (13) and solving the resulting quadratic equation we obtain $g_{m}:$

$$
\begin{aligned}
g_{m} & =\frac{g_{i} N_{j}-g_{j} N_{j}-3 g_{i} N_{w}-g_{j} N_{w}}{2 N_{t}}+ \\
& +\frac{1}{2 N_{t}} \sqrt{12 g_{i} g_{j}\left(N_{j}-N_{w}\right)\left(3 N_{j}+N_{w}\right)+\left(g_{i}\left(N_{j}-3 N_{w}\right)-g_{j}\left(N_{j}+N_{w}\right)\right)^{2}}
\end{aligned}
$$

It is worth mentioning that if one wishes to work with wire density $\left(n_{w}\right)$ and junction density $\left(n_{j}\right)$ instead of total numbers the form of the above expressions remains the same. Simply set $N_{j} \rightarrow n_{j}$ and $N_{w} \rightarrow n_{w}$. It is possible to relate the wire density with junction density; Heitz et al. 26] found that for a given wire density $n_{w}$ of identical wires of length $L$, the junction density is given by

$$
n_{j}=\frac{1}{2} P \pi L^{2} n_{w}^{2}
$$

where $P$, the contact probability, was calculated numerically by Heitz et al. and found to be 0.2027 . The wire lengths of our experimental NWN samples mostly follow a Gaussian distribution centered on $6.7 \mu \mathrm{m}$. Subbing this value in to Eq. (21) one finds that $n_{j} \approx$ $15.6 n_{w}^{2}$. However results of simulations for wire lengths that follow a Gaussian distribution gives the relation $n_{j} \approx 13 n_{w}^{2}$ or $n_{j} \approx 0.265 L^{2} n_{w}^{2}$. From here on we shall use the notation 
$n_{j}=\alpha(L) n_{w}^{2}$ with $\alpha \approx 13$. Thus, in terms of wire density alone Eq. (20) becomes

$$
\begin{aligned}
g_{m} & =\frac{g_{i} \alpha n_{w}-3 g_{i}-g_{j}-g_{j} \alpha n_{w}}{2\left(3 \alpha n_{w}+1\right)}+ \\
& +\frac{1}{2\left(3 \alpha n_{w}+1\right)} \sqrt{12 g_{i} g_{j}\left(\alpha n_{w}-1\right)\left(3 \alpha n_{w}+1\right)+\left(g_{i}\left(\alpha n_{w}-3\right)-g_{j}\left(\alpha n_{w}+1\right)\right)^{2}}
\end{aligned}
$$

and provides a closed-form expression that maps the conductance of a disordered NWN with that of a ordered homogeneous lattice composed of edge resistors of resistances $R=1 / g_{m}$. As repeatedly mentioned in this manuscript, the agreements displayed in Figures 2 and 3 were not coincidental nor were they generated through a fitting scheme. They were in fact obtained with the use of Eq. (22).

\section{RESULTS}

In addition to the results seen in Figs. 2 and 3 , where the sheet resistance is shown as a function of electrode separation, our method is also capable of demonstrating how the resistance depends on the wire density. With that in mind, we consider disordered NWNs that were either randomly generated through a simulation or were image processed and corresponded to experimentally deposited networks (see Figs. 1(a) and 1(b)) [13].

Simulations involved generating NWN with resistances that follow the distributions outlined above. Networks of physical size $\mathrm{W} \times \mathrm{W}$ and wire density $n_{w}$ are then contacted by finite-sized electrodes also of size W, as shown in Fig. 3(a). The inter-electrode resistance is recorded and averaged over. The number of nodes along each electrode $\left(N_{y}\right)$ and the path length between electrodes of each sample $\left(N_{x}\right)$ can be calculated using methods outlined in Appendix A. We recall that, following Eq. (12), the value of $R$ alone is not sufficient to calculate the sheet resistance, but depends on the fraction $\frac{N_{x}}{N_{y}}$ as well. Eq. 12 becomes

$$
R_{e}\left(n_{w}\right)=\frac{R\left(n_{w}\right) \pi C}{l^{2} n_{w} P_{i}} \log \left(6 l^{2} \alpha n_{w} P_{i}\right)
$$

where $\mathrm{C}$ is a constant and $P_{i}$ is defined in Eq. (15). In Figure 5 the sheet resistance is shown as a function of the wire density. Once again, points correspond to the numerically evaluated results with the error bars indicating the fluctuations that arise each time a new wire configuration is considered. The solid line indicates the effective-medium result obtained through Eq. (23). Two scaling regions can be identified in Fig. 5(a), namely $n_{w} \lesssim 0.25$ and $n_{w} \gtrsim 0.25$. In the low-density range, the sheet resistance has a varying scaling behaviour 


\section{(a)}
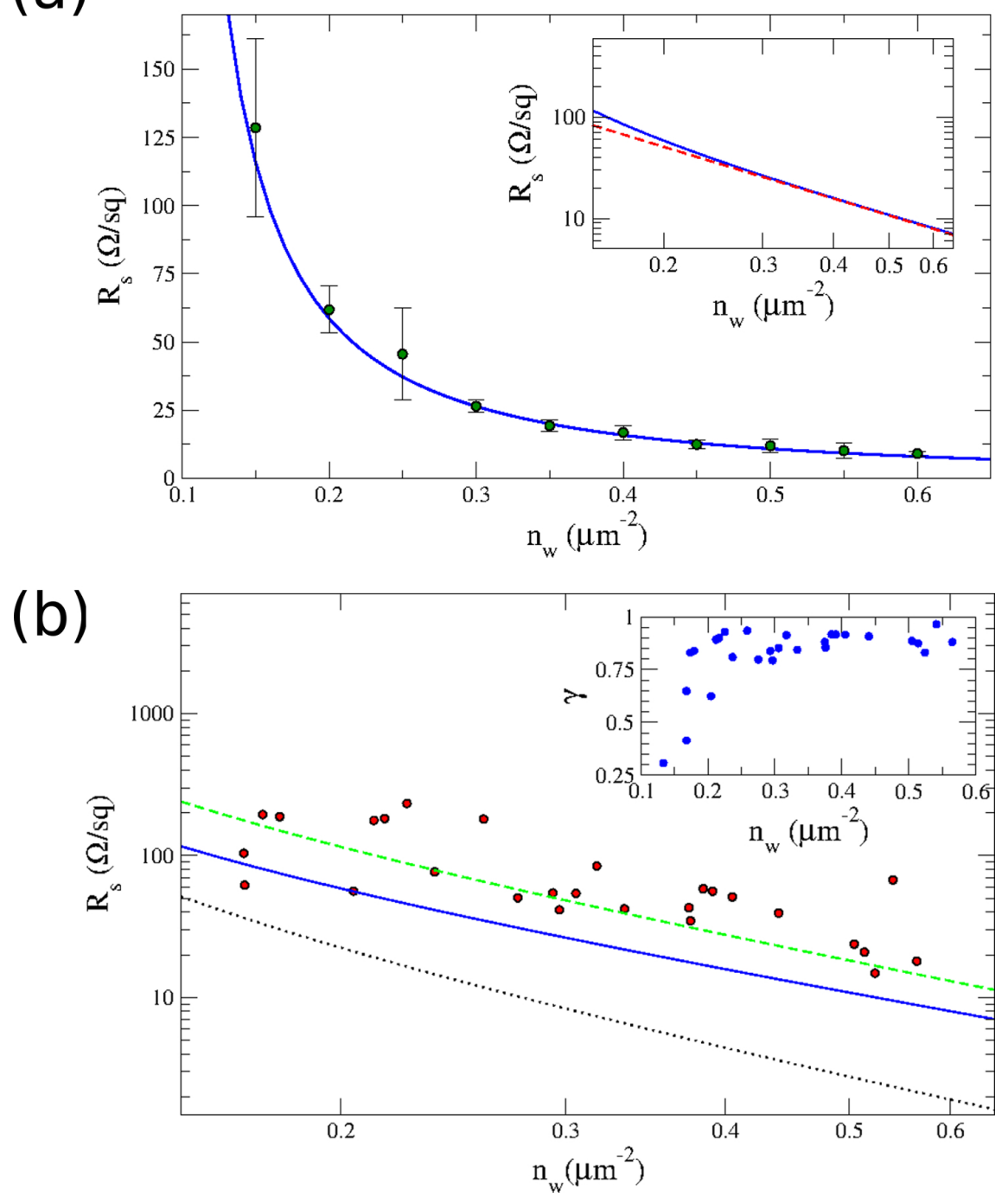

FIG. 5: In figure (a), data points show the sheet resistance for a given wire density, averaged over a number of spatial configurations. The solid line represents the dependence of Eq. 23) on $n_{w}$. The inset figure shows that theory converges to a power law $\propto n_{w}^{-1.7}$. Figure $(b)$ is a comparison of experimental sheet resistance and values determined by Eq. (23). The data points correspond to experimental measurements on Ag NWNs that have been reported in our previous publication [13]. The solid line is the sheet resistance given when there are no outliers included in the Junction resistance distribution. The dashed line is when $10 \%$ of junction resistors are outliers. The dotted line shows $R_{0}$ as a function of wire density. $R_{0}$ is the sheet resistance when every junction has 0 resistance.

but converges to a power law $\propto n_{w}^{-1.7}$ in the latter region. The top panel shows results for computer-generated NWNs, with an inset depicting the same results in logarithmic scale. The inset indicates the appearance of a clear power-law behaviour when the density increases. The dashed line of slope -1.7 serves as a guide to the eyes and highlights the exponent of the said power law. It is worth pointing out that the size of the error bars is much smaller in 
the high-density region $\left(n_{w} \gtrsim 0.25\right)$ since the fluctuations resulting from spatial dispersion of the wires on the network area are smaller in this range.

Finally, instead of computer-generated networks, we made use of experimentally deposited Ag NWN samples that were image processed in order to generate the location and orientation of all wires in the network. Samples are the same as reported in our recent publication [13]. Values of $N_{x}$ and $N_{y}$ are taken from simulations of NWNs of the same size as the samples $(\sim 20 \mu \mathrm{m} \times 20 \mu \mathrm{m})$. It is important to highlight a fundamental difference between the computer-generated simulations and the experimentally deposited samples. In the former, besides having full information about the wire locations, we also have access to the assigned junction resistances. In the latter, while we also have information about the spatial distribution of the wires after the image processing is carried out, we can only infer about the actual junction distribution for a given sample. We make use of the recently measured distribution [15] of junction resistances shown in Fig. 4 with the assumption that this distribution is representative of all NWNs.

As shown in Fig. 4, a continuous distribution for the junction resistance can be found up to $75 \Omega$ with a few outliers (junctions with characteristic resistances of $R>200 \Omega$ ) located at high-resistance range. The existence of these high-resistance outliers in the distribution is associated with wires that were not electrically stressed and are still dormant, not taking active part in the transport of electric current. Bellew et al. [15] argued that no matter how strongly the network is electrically stressed, there will always be a small percentage of these high-resistance junctions. Because our effective model depends crucially on the resistance distributions, as seen in Eq. (19), whether or not these high-resistance outliers are included in the junction-resistance distribution is expected to have an impact on our results. Indeed, Fig. 5(b) clearly indicates that the agreement between the numerically evaluated results and those obtained by the effective medium improves after such high-resistance outliers are included in the distribution.

Another use for the effective-medium theory presented here is that we can estimate how close (or far) a particular sample is from operating at its minimum resistance state $R_{0}$. The optimization-capacity coefficient $\gamma$ was recently introduced and it quantifies how much room for improvement a given network has for reducing its sheet resistance upon electrical stress. [13] It is defined as $\gamma=1-R_{0} / R_{\text {exp }}$, where $R_{\text {exp }}$ is the experimentally measured resistance and $R_{0}$ is the resistance that the network would have if all interwire contacts were 
perfect. Values of $\gamma$ close to 1 represent networks whose conductivities can be considerably improved since their sheet resistances are far from the optimal value $R_{0}$. For relatively small values of $\gamma$, on the other hand, the network is close to its optimum conductivity and is unlikely that it can be further optimized. In reference [13], the value of $R_{0}$ was obtained through numerical simulations that involved solving the complete set of Kirchhoff's circuit equations. A much faster way of obtaining the same quantity is to use the effective-medium theory to find the optimal resistance by simply adapting the distribution in Eq. (19) to the characteristics of real NWN samples. The dotted line in Fig. 5(b) displays $R_{0}$ as a function of the wire density. It is evident in this graph that the discrepancy between the experimental points and the optimal resistance $R_{0}$ increases with the wire density. This is further confirmed by the inset of Fig. 5(b) showing how the optimization-capacity coefficient depends on the the wire density. Besides proving to be a very efficient way of assessing how much scope for optimization a given sample may have, this also indicates that the junctionresistance contribution is less important for low-density networks. This can be explained by the fact that the spacing between nodes on the same wire is inversely proportional to $n_{w}$, which causes $R_{0}$ to increase as the network becomes more sparse. Therefore, the greater $R_{0}$ the closer it gets to the experimentally measured values, which in turn gives less scope for optimization.

\section{CONCLUSION}

In summary, we have outlined a simple method that establishes the correspondence between the sheet resistance of a heavily disordered NWN with that of an ordered network. In doing so we provided a closed-form expression for the effective conductance of the NWN that reflects the setup commonly seen in real experiments of this type, i.e., finite-sized electrodes embedded into a network made of wires with a distribution of physical characteristics (length, diameter, resistivity, etc). It is important to stress that this is done without the need of any fitting scheme. Excellent agreement with both computer-generated and experimentally grown networks was observed. Besides identifying how the sheet resistance of disordered NWN depends on the wire density, we can assess in a far more efficient way how relevant the intra-wire resistivity is in determining the overall sheet resistance of the network. This framework can be easily generalized to investigate how the sheet resistance of 
NWNs changes with a range of other parameters and it proves to be very useful in guiding further experimental attempts to optimize the conductivity of NWN films.

\section{Acknowledgments}

The authors wish to acknowledge funding from the European Research Council (ERC) under Advanced Grant 321160. This publication has emanated from research supported in part by a research grant from Science Foundation Ireland (SFI) under Grant Number SFI/12/RC/2278. The support of TCHPC at Trinity College Dublin for computational resources is also acknowledged.

\section{APPENDIX}

Consider a wire of length $L$. If the center point of the wire is a distance $x<\frac{L}{2}$ from an electrode, the two will intersect if the angle $\theta$ is in the range

$$
\theta \leq \cos ^{-1}\left(\frac{2 x}{L}\right)
$$

where $\theta$ is the angle the wire makes with the horizontal.

A wire at a distance $x$ intersects the electrode with a probability $\frac{2}{\pi} \theta$. In order to obtain a probability that a wire intersects the $y$ axis once its center is $x \leq \frac{L}{2}$ we perform an integration over $x$ :

$$
\frac{2}{\pi} \int_{0}^{1} d x \cos ^{-1}(\theta)=\frac{2}{\pi}
$$

We now consider how many wires lie in the range that they could potentially intersect the electrode. If wires are distributed homogeneously with a density of $n_{w}$ and over a vertical width range of $\mathrm{W}$, the relevant area is $W L / 2$ which contains $W L n_{w} / 2$ wires. Combining this with the value of $p_{e}$, the expected total number $\left(N_{y}\right)$ of intersections can be written as

$$
N_{y}=\frac{L W n_{w}}{\pi}
$$

A Watts-Strogatz (WS) network is an example of a small-world network [27]. A WS network is created by taking a regular lattice network where each node has $z$ nearest neighbours. A percentage $p$ of links are removed and are then used to connect random pairs of nodes. Braunstein et al[28] showed that when weak disorder is introduced to the weight 
values of links the optimal path $q_{\text {opt }}$ that minimises the total weight of the path connecting two nodes scales as:

$$
q_{\text {opt }} \propto \frac{1}{p z^{2}} \log (N p z)
$$

Where $\mathrm{N}$ is the number of nodes in the network.

We assume that NWNs of size $l \times l$ behave as small-world networks, where $l$ is the typical length of a NW. In our model the number of nodes in the network is $2 N_{j}$. Each node is connected to one junction resistor, a wire segmant and either another wire segmant or a dead end. The degree of each node is thus $z=3$. $p$ is taken to be the percentage of current carrying intra-wire segments in the network as they can connect two nodes that have a large separation. Therefore $p=P_{i}=\frac{2 N_{j}-N_{w}}{3 N_{j}+N_{w}}$ from section II.B. Subbing this into Eq 27, $q_{\text {opt }}$ scales as

$$
q_{\text {opt }} \propto \frac{1}{P_{i}} \log \left(6 N_{j} P_{i}\right)=\frac{3 N_{j}+N_{w}}{2 N_{j}-N_{w}} \log \left(6 N_{j} \frac{2 N_{j}-N_{w}}{3 N_{j}+N_{w}}\right)
$$

Consider a network of size $W \times W, W>>L$ and node $\mathrm{A}$ that lies on the electrode of the NWN. The optimal path between node A and node B that are separated by a distance $q_{\text {opt }}$ defined above. Similarly the distance between node B and another node $\mathrm{C}$ that are again separated by a distance $q_{o p t}$ and so on. Therefore the optimal path between the two electrodes, $N_{x}$ is found by multiplying Eq.28 by a prefactor of $\frac{W}{L}$. Thus

$$
N_{x}=\frac{W}{L} \frac{C}{P_{i}} \log \left(6 N_{j} P_{i}\right)
$$

where $\mathrm{C}$ is a constant $\approx 1$.

[1] J. Liang, L. Li, K. Tong, Z. Ren, W. Hu, X. Niu, Y. Chen, and Q. Pei, ACS Nano 8, 1590 (2014), pMID: 24471886, http://dx.doi.org/10.1021/nn405887k, URL http://dx.doi.org/ $10.1021 / \mathrm{nn} 405887 \mathrm{k}$.

[2] S. M. Bergin, Y.-H. Chen, A. R. Rathmell, P. Charbonneau, Z.-Y. Li, and B. J. Wiley, Nanoscale 4, 1996 (2012), URL http://dx.doi.org/10.1039/C2NR30126A.

[3] D. Langley, G. Giusti, C. Mayousse, C. Celle, D. Bellet, and J.-P. Simonato, Nanotechnology 24, 452001 (2013), URL http://stacks .iop.org/0957-4484/24/i=45/a=452001. 
[4] M. Song, D. S. You, K. Lim, S. Park, S. Jung, C. S. Kim, D.-H. Kim, D.-G. Kim, J.-K. Kim, J. Park, et al., Advanced Functional Materials 23, 4177 (2013), ISSN 1616-3028, URL http://dx.doi.org/10.1002/adfm.201202646.

[5] D. Angmo, T. R. Andersen, J. J. Bentzen, M. Helgesen, R. R. Sndergaard, M. Jrgensen, J. E. Carl, E. Bundgaard, and F. C. Krebs, Advanced Functional Materials 25, 4539 (2015), ISSN 1616-3028, URL http://dx.doi.org/10.1002/adfm.201501887.

[6] A. Kim, Y. Won, K. Woo, C.-H. Kim, and J. Moon, ACS Nano 7, 1081 (2013), pMID: 23330971, http://dx.doi.org/10.1021/nn305491x, URL http://dx.doi.org/10.1021/ nn305491x.

[7] Y.-J. Choi, I.-S. Hwang, J.-G. Park, K. J. Choi, J.-H. Park, and J.-H. Lee, Nanotechnology 19, 095508 (2008), URL http://stacks .iop.org/0957-4484/19/i=9/a=095508.

[8] N. Datta, N. S. Ramgir, S. Kumar, P. Veerender, M. Kaur, S. Kailasaganapathi, A. Debnath, D. Aswal, and S. Gupta, Sensors and Actuators B: Chemical 202, 1270 (2014), ISSN 09254005, URL http://www.sciencedirect.com/science/article/pii/S0925400514007618.

[9] A. R. Madaria, A. Kumar, F. N. Ishikawa, and C. Zhou, Nano Research 3, 564 (2010), ISSN 1998-0000, URL http://dx.doi.org/10.1007/s12274-010-0017-5.

[10] B. Hwang, H.-A.-S. Shin, T. Kim, Y.-C. Joo, and S. M. Han, Small 10, 3397 (2014), ISSN 1613-6829, URL http://dx.doi.org/10.1002/smll.201303906.

[11] L. Hu, H. S. Kim, J.-Y. Lee, P. Peumans, and Y. Cui, ACS Nano 4, 2955 (2010), pMID: 20426409, http://dx.doi.org/10.1021/nn1005232, URL http://dx.doi.org/10.1021/ nn1005232,

[12] P. N. Nirmalraj, A. T. Bellew, A. P. Bell, J. A. Fairfield, E. K. McCarthy, C. OKelly, L. F. C. Pereira, S. Sorel, D. Morosan, J. N. Coleman, et al., Nano Letters 12, 5966 (2012), pMID: 23062152, http://dx.doi.org/10.1021/nl303416h, URL http://dx.doi.org/10.1021/ nl303416h,

[13] C. Rocha, H. Manning, C. OCallaghan, C. Ritter, A. Bellew, J. Boland, and M. Ferreira, Nanoscale 7, 13011 (2015).

[14] R. M. Mutiso, M. Sherrott, A. Rathmell, B. Wiley, and K. Winey, ACS Nano 7, 7654 (2013).

[15] A. Bellew, H. Manning, C. Rocha, M. Ferreira, and J. Boland, ACS Nano 9, 11422 (2015).

[16] G. E. Pike and C. H. Seager, Phys. Rev. B 10, 1421 (1974), URL http://link.aps.org/ doi/10.1103/PhysRevB.10.1421 
[17] J. Li and S. Zhang, Phys. Rev. E 80, 040104 (2009).

[18] M. Žeželj and I. Stanković, Phys. Rev. B 86, 134202 (2012), URL http://link.aps.org/ doi/10.1103/PhysRevB.86.134202.

[19] A. Behnam and A. Ural, Phys. Rev. B 75, 125432 (2007), URL http://link.aps.org/doi/ 10.1103/PhysRevB.75.125432.

[20] J. Cserti, Am. J. Phys. 68, 896 (2000).

[21] E. N. Economou, Green's functions in quantum physics, vol. 3 (Springer, 1984).

[22] S. P., Scattering and localization of classical waves in random media (World Scientific, 1990).

[23] S. Kirkpatrick, Rev. Mod. Phys. 45, 574 (1973).

[24] C. Pozrikidis, An Intrduction to Grids, Graphs and Networks (Oxford University Press, 2014).

[25] J. van de Groep, P. Spinelli, and A. Polman, Nano Letters 12, 3138 (2012), pMID: 22554260, http://dx.doi.org/10.1021/nl301045a, URL http://dx.doi.org/10.1021/nl301045a.

[26] J. Heitz, Y. Leroy, L. Hbrard, and C. Lallement, Nanotechnology 22, 345703 (2011), URL http://stacks.iop.org/0957-4484/22/i=34/a=345703.

[27] D. J. Watts and S. H. Strogatz, nature 393, 440 (1998).

[28] L. A. Braunstein, S. V. Buldyrev, R. Cohen, S. Havlin, and H. E. Stanley, Physical review letters 91, 168701 (2003). 